\title{
ArcheoSciences
}

Revue d'archéométrie

$36 \mid 2012$

Varia

\section{L'or de la vallée de la Somme : recherches sur le monnayage d'or ambien (IIIe-Ier siècle av. J.-C.)}

The Somme valley's gold: Research on Ambiani's gold coinage

Charlotte Sillon, Sylvia Nieto-Pelletier et Bernard Gratuze

\section{OpenEdition}

1 Journals

Édition électronique

URL : https://journals.openedition.org/archeosciences/3819

DOI : 10.4000/archeosciences.3819

ISBN : 978-2-7535-2243-5

ISSN : $2104-3728$

Éditeur

Presses universitaires de Rennes

Édition imprimée

Date de publication : 31 décembre 2012

Pagination : 117-126

ISBN : 978-2-7535-2241-1

ISSN : $1960-1360$

\section{Référence électronique}

Charlotte Sillon, Sylvia Nieto-Pelletier et Bernard Gratuze, "L'or de la vallée de la Somme : recherches sur le monnayage d'or ambien (IIle-ler siècle av. J.-C.) », ArcheoSciences [En ligne], 36 | 2012, mis en ligne le 31 décembre 2014, consulté le 01 février 2022. URL : http://journals.openedition.org/ archeosciences/3819; DOI : https://doi.org/10.4000/archeosciences.3819 


\title{
L'or de la vallée de la Somme : recherches sur le monnayage d'or ambien (III $-\mathrm{I}^{\mathrm{er}}$ siècle av. J.-C.)

\author{
The Somme Valley's Gold: Research on Ambiani's Gold Coinage
}

\author{
CharlotteS ILlon*,S ylviaN eto-Pelletier ${ }^{* *}$ et B ernardG RATUZE**
}

\begin{abstract}
Résumé : C’est dans la vallée de la Somme qu’ont été frappées les premières émissions monétaires de Gaule Belgique, exclusivement en or. Elles sont à l'origine d'un monnayage d'or qui perdure de manière continue jusqu'au I ${ }^{\text {er }}$ siècle avant J.-C. Au cours des différentes phases de son histoire, son aire de circulation ne cesse de s'élargir pour englober finalement l'ensemble de la Gaule Belgique et le sud de la Bretagne Insulaire. La compréhension des enjeux propres à ce monnayage s'enrichit désormais de l'apport des données relatives à la composition élémentaire des alliages monétaires. Ces données nouvelles ont permis de vérifier les données typologiques et métrologiques, en mettant en évidence trois phases distinctes du point de vue de la composition monétaire : la première témoigne de l'usage, pour la frappe monétaire, d'un or natif, avec ou sans ajout de cuivre. Dans un second temps, avec la mise en circulation des statères au flan large, les autorités émettrices introduisent le procédé de l'altération de l'alliage monétaire par l'ajout d'argent et de cuivre, dans des proportions encore limitées. Enfin, au I ${ }^{\text {er }}$ siècle avant J.-C., la fabrication monétaire est plus strictement contrôlée et l'altération de l'alliage devient importante et systématique. En outre, les analyses révèlent la variété des techniques employées dans la fonte et la frappe des monnaies.

Abstract: The Somme valley is the birthplace of the Celtic coins of Belgic Gaul, exclusively made of gold. From those emissions derived a range of gold coinages emitted until the It century B.C. Their distribution kept on widening, eventually encompassing Belgic Gaul as a whole, as well as southern Britain. The new data made available about its metallic composition enables one to better understand the issues of this peculiar coinage. The analysis has indeed emphasized three successive phases in relation with metallic composition, which are consistent with the typological and metrological data. During the first stages of the process, the alloy is clearly derived from native gold, containing various amounts of copper. Then, the "broad flan " series can be regarded as a transitional phase, during which the alloy is altered by adding silver and copper altogether, although in a limited proportion. Finally, during the Ist century B.C., such techniques are widely used, while the whole manufacturing process is more strictly controlled. Furthermore, the analysis reveals the wide variety of technical solutions which have been used for alloy melting and coinage striking.
\end{abstract}

Mots clé : numismatique, Gaule Belgique, LA-ICP-MS, or.

Keywords: numismatics, Belgic Gaul, LA-ICP-MS, gold.

\section{INTRODUCTION}

La vallée de la Somme joue un rôle prépondérant dans l'histoire monétaire celtique puisque c'est dans cette région qu'ont été frappées les premières monnaies de Gaule Belgique, exclusivement en or. La vallée de la Somme est occupée à partir du IV siècle avant J.-C. par le peuple des Ambiens (Kruta, 2000). L'étude du monnayage d'or attribué à ce peuple permet de mieux comprendre la naissance de la monnaie en Gaule Belgique et de retracer les étapes de son évolution jusqu’à la guerre des Gaules.

* Doctorante en histoire avec une bourse de la Région Centre, sous la direction de Bernard GRATUZE, Stephan FICHTL et le co-encadrement de Sylvia NIETO-PELLETIER, IRAMAT, Centre Ernest-Babelon, UMR 5060, CNRS - Université Orléans; charlotte.sillon@gmail.com

** Chargée de recherche, IRAMAT, Centre Ernest-Babelon, UMR 5060, CNRS - Université Orléans. (nieto@cnrs-orleans.fr) (gratuze@cnrs-orleans.fr) 
Les monnaies de l'ouest de la Gaule Belgique ont fait l'objet d'un important travail de classement typologique par Simone Scheers (Scheers, 1977), mis à jour plus récemment par John Sills (Sills, 2003). Ces différentes études ont permis de mettre en évidence une chronologie relative des différentes émissions attribuées aux Ambiens, qui se fonde sur l'observation d'une simplification progressive du type monétaire : le style grec affirmé des premières monnaies laisse progressivement place à une iconographie celtisée, de plus en plus schématique au fil des émissions, jusqu'à disparaitre du droit (tableau 1).

Les analyses réalisées dans le cadre des études des monnayages de l'ouest de la Gaule Celtique publiées dans les Cahiers Ernest-Babelon sous la direction de Jean-Noël Barrandon (Barrandon, 1994), valident le principe de l'altération continue des monnayages en or. Cet ajout volontaire d'argent et de cuivre est alors utilisé comme un indicateur d'une chronologie relative des émissions. Cette théorie est ensuite appliquée à plusieurs monnayages de Gaule Celtique (Scheers, Barrandon, 1994; Aubin, Barrandon, 1994; Nieto, 2003). Un des objectifs de la présente étude portant sur les monnaies d'or de la vallée de la Somme est d'associer aux classements typologiques existants les données analytiques afin de compléter la connaissance de ce monnayage et de tenter de confirmer, ou d'infirmer, la chronologie relative précédemment mise en place.

L'analyse élémentaire des monnaies étudiées s'est déroulée en deux temps. Entre 1998 et 2003, Jean-Noël Barrandon a analysé 40 monnaies d'or belges par activation protonique, dont 26 sont attribuées aux Ambiens. Plus récemment, 47 autres exemplaires ainsi que deux statères de Tarente ont été analysés par spectrométrie de masse à plasma avec micro-prélèvement par ablation laser. Il s'agit d'une méthode d'analyse multi-élémentaire séquentielle à la fois qualitative et quantitative, qui permet de doser les trois principaux éléments constitutifs des alliages à base d'or (or, argent, cuivre), ainsi que la plupart des impuretés naturellement présentes dans ces métaux, soit 18 éléments, jusqu'à l'échelle de la partie par million (ppm). Les objets étudiés sont placés à l'intérieur d'une cellule traversée par un flux d'argon. Un micro-prélèvement, invisible à l'œil nu, est effectué par un rayon laser. Le diamètre

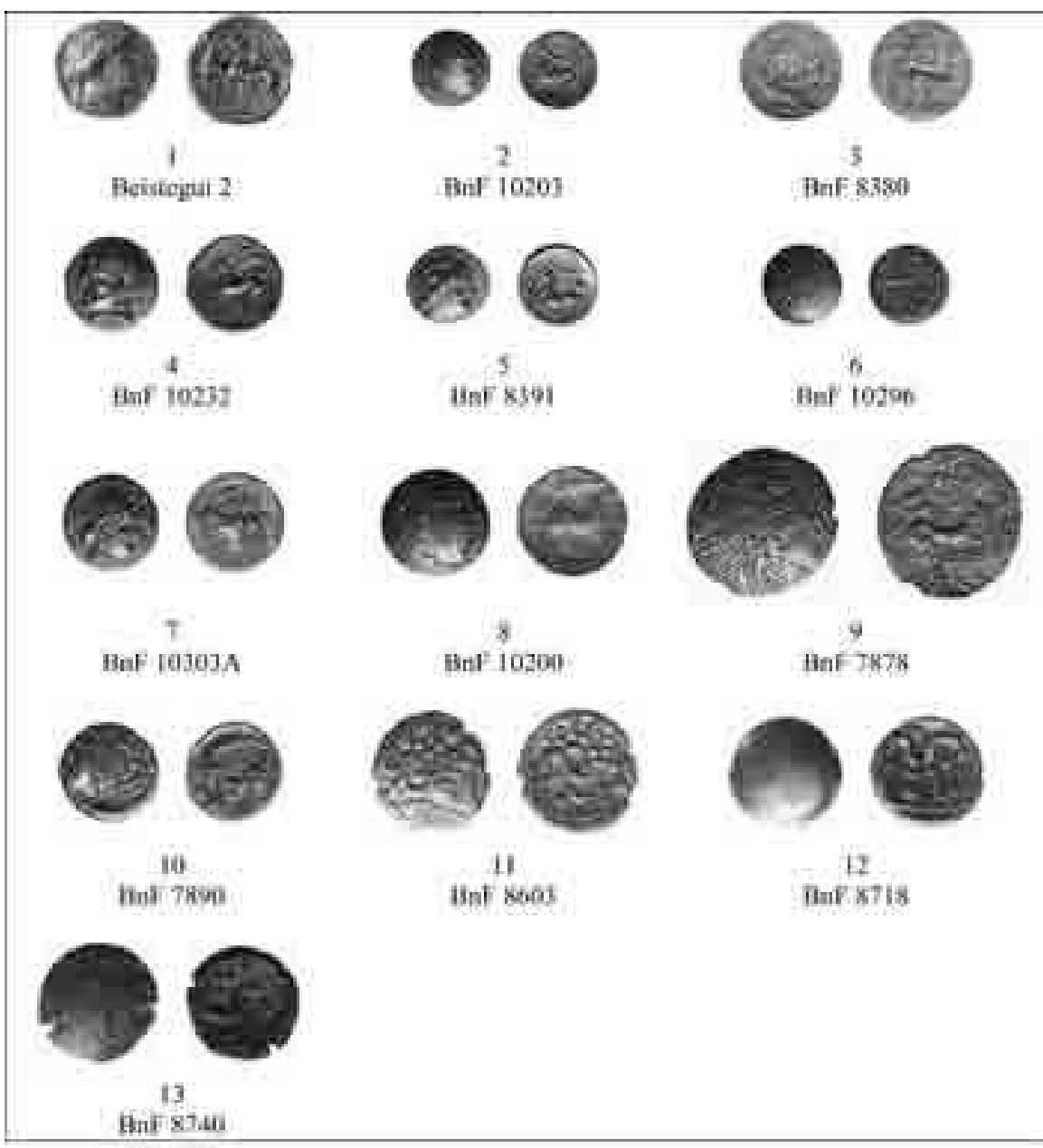

Tableau 1 : (Voir planche couleur) Planche de monnaies.

Table 1: (See colour plate) Coin plate. 
de l'impact est compris entre 0,02 et $0,08 \mathrm{~mm}$, sa profondeur dépend de la durée de l'ablation et peut atteindre $1 \mathrm{~mm}$. Cette durée est en fait adaptée au matériau étudié et vise à atteindre une zone de composition stable à l'intérieur de l'objet. La matière prélevée est ensuite transportée vers une torche à plasma par le flux d'argon où elle est dissociée et ionisée. Les différents constituants sont identifiés selon leur masse grâce à un secteur magnétique couplé à un secteur électrostatique, puis quantifiés par un détecteur de type channeltron associé à une cage de Faraday. Un système informatique récupère les données et permet le calcul de la composition. Deux microprélèvements sont réalisés pour chaque analyse. La composition de l'objet analysé est obtenue en utilisant un programme informatique, développé au laboratoire, qui fait appel à une méthode originale de calcul par étalonnage interne. Une adaptation récente de cette méthode permet de calculer la composition de l'objet au fur et à mesure de la pénétration du laser. Cette approche permet à la fois d'obtenir les teneurs des différents éléments de la surface de l'objet, souvent perturbée par des traitements métallurgiques et/ou la corrosion, mais aussi au cœur de celui-ci, zone qui correspond à celle de l'alliage travaillé. Cette mesure en profil de concentrations concerne tous les éléments, majeurs, mineurs et traces. On peut ainsi vérifier l'homogénéité de l'alliage employé, pour l'ensemble de ses constituants. La précision des mesures varie selon la nature et l'homogénéité des alliages étudiés (tableau 2). Cette méthode présente l'avantage de réaliser l'analyse rapidement et permet de rendre les monnaies à leur institution de conservation dans des délais brefs. (Gratuze, Blet-Lemarquand, Barrandon, 1994)

\section{L'APPARITION DU MONNAYAGE D'OR Dans la Vallée de la Somme}

De la même manière que dans les autres territoires gaulois, les premières monnaies frappées dans la vallée de la Somme sont influencées par la typologie et la métrologie des monnayages méditerranéens. En effet, la première série monétaire frappée dans cette région est une imitation fidèle

\begin{tabular}{|c|c|c|}
\hline Elemem ariabut & Inmint de odtertibsi tonm! & Tcart troe ist \\
\hline ais & nens & - \\
\hline As & 4 & $a, 5$ \\
\hline Sy & 3 & 78 \\
\hline 10 & 6,00 & 1.8 \\
\hline n & Q.e1 & 4 \\
\hline $\mathrm{Pe}$ & 003 & 3.5 \\
\hline
\end{tabular}

Tableau 2 : Éléments analysés par LA-ICP-MS.

Table 2: Elements analysed by LA-ICP-MS. d'un statère de Tarente, présentant au droit une tête d'Héra voilée, au revers les Dioscures (tableau 1; 1). Il existe parallèlement à cette émission des imitations du statère de Philippe II de Macédoine, qui se caractérise par une tête d'Apollon lauré au droit et un bige au revers. Plusieurs séries se distinguant par des éléments typologiques découlent ensuite de ces deux émissions originelles (tableau 1 ; 3-8). La gravure de ces premières monnaies est fine et proche de celle des monnaies grecques. D'un point de vue métrologique, ces séries comprennent des monnaies d'environ 4 grammes (g) et $2 \mathrm{~g}$, ce qui correspond aux poids des hémistatères et des quarts de statère grecs.

Les compositions élémentaires des 26 exemplaires analysés forment deux groupes distincts (fig. 1). Le premier présente un titre moyen de $94,0 \%( \pm 2,0)$, des teneurs en argent peu élevées, en moyenne $4,2 \%( \pm 1,9)$, et des teneurs en cuivre faibles, de $1,5 \%$ au maximum. Nous reconnaissons ici les caractéristiques de l'or natif, n'ayant subi ni purification, ni altération. Le deuxième groupe, pour sa part, est composé d'exemplaires présentant un titre plus variable compris entre $49,8 \%$ et $87,7 \%$, des teneurs en argent fluctuant entre $10,6 \%$ et $49,8 \%$ et des valeurs en cuivre d'une moyenne de 2,8 \% $( \pm 2,9)$. Il ne s'agit pas forcément d'un ajout volontaire d'argent, dans la mesure où cet élément est naturellement présent dans le minerai aurifère, dans des proportions variables pouvant atteindre $40 \%$ (Barrandon, 1994); en revanche, les teneurs en cuivre ne peuvent pas être expliquées de la même manière. En effet, les travaux de Robert Chapman (Chapman, 2006) ont permis de mettre en évidence la quasi absence de cuivre $(<1 \%)$ dans le minerai aurifere, ce qui nous porte à conclure que la composition des monnaies du second groupe révèle un ajout de cuivre. Toutefois, dans la mesure où les teneurs sont d'une moyenne de 2,8 \%, l'hypothèse classique d'une volonté d'abaissement de la teneur en or ne parait pas convaincante. Dès lors, deux possibilités peuvent être envisagées : il peut s'agir d'un ajout volontaire motivé par une logique technique, le cuivre ayant un impact positif sur la dureté de l'alliage; inversement, on peut envisager un ajout accidentel, lié par exemple à la réutilisation d'un creuset lors du processus de fonte, ou à un approvisionnement différent en or (refonte de bijoux...). Cette dernière hypothèse semble confortée par les variations de la composition de l'alliage au sein d'une même série, comme dans le cas de celle à la tête barbue.

Les premières monnaies d'or de la vallée de la Somme imitent des statères d'or grecs de Tarente et aux types de Philippe II de Macédoine. Au-delà de cette imitation d'ordre typologique, la question se pose de déterminer si les autorités émettrices se sont aussi alignées sur les compositions élémentaires des prototypes. À cet effet, plusieurs analyses 


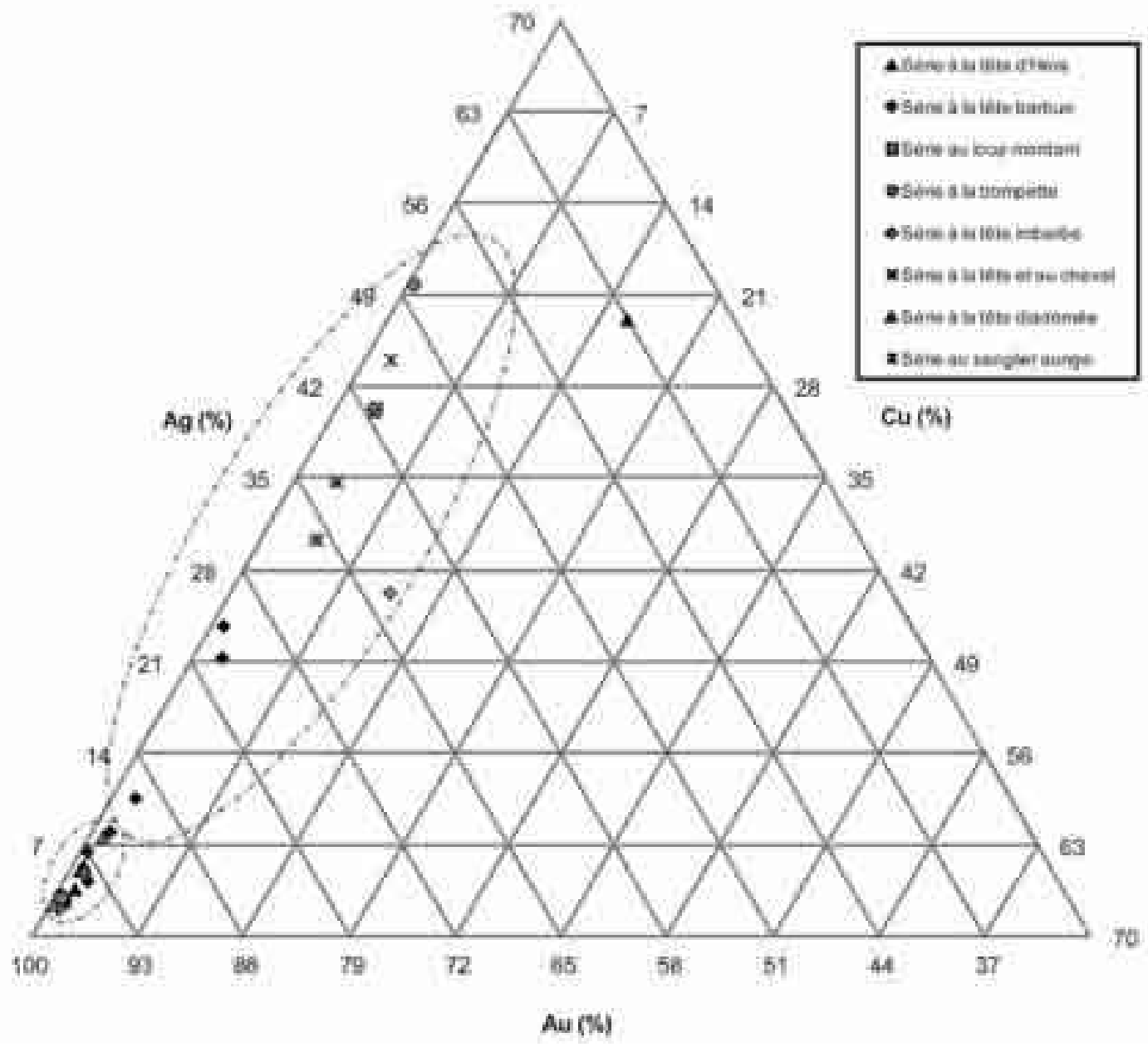

Figure 1 : composition élémentairs des premières émissions en or frappées dans la vallée de la Somme.

Figure 1: elementary composition of the first gold series struck in the Somme valley.

ont été effectuées pour permettre une comparaison entre ces différentes émissions, grecques et belges. On constate alors, qu'à l'or natif employé pour les premiers monnayages de la vallée de la Somme, s'oppose un or purifié, composé de moins de $1 \%$ d'argent et $1 \%$ de cuivre dans le cas des statèresg recs( fig. 2).

Concernant les premières émissions de la vallée de la Somme, il apparait donc qu'à un premier groupe frappé à base d'or natif s'ajoute un deuxième groupe dont l'alliage est marqué par un faible ajout de cuivre. Dans la mesure où les variations au sein d'un même gisement d'or peuvent être importantes, l'utilisation d'un or natif n'offre aucune garantie quant à la constance du titre. De ce fait, étant données les variations observées, on peut supposer qu'il n'était pas contrôlé par les autorités émettrices, tandis que d'autres critères d'ordre métrologique, tels que le poids ou le diamètre, pourraient avoir été retenus.

\section{DÉVELOPPEMENT DE LA FRAPPE MONÉTAIRE : LA SÉRIE AU FLAN LARGE}

Le volume des monnaies en circulation semble augmenter à partir de la série au flan large (tableau $1 ;$ 9-10). D'autres évolutions se font également jour quant aux caractéristiques de cette série : en premier lieu, on observe l'ajout d'une nouvelle dénomination, le statère. Les flans sont exceptionnellement grands avec, pour les statères, un diamètre variable allant jusqu'à 26 millimètre $(\mathrm{mm})$, tandis que le diamètre des quarts de statère reste stable à $14 \mathrm{~mm}$. Les statères pèsent en moyenne $7,50 \mathrm{~g}$ et le poids des quarts de statère chutent à $1,80 \mathrm{~g}$. La typologie s'est éloignée du style grec des premières monnaies pour faire place à une iconographie celtisée. La frappe monétaire s'intensifie et la circulation de cette série s'élargit. Les cartes de répartition pour ces émissions mettent en avant une circulation dans tout l'ouest de la Gaule 


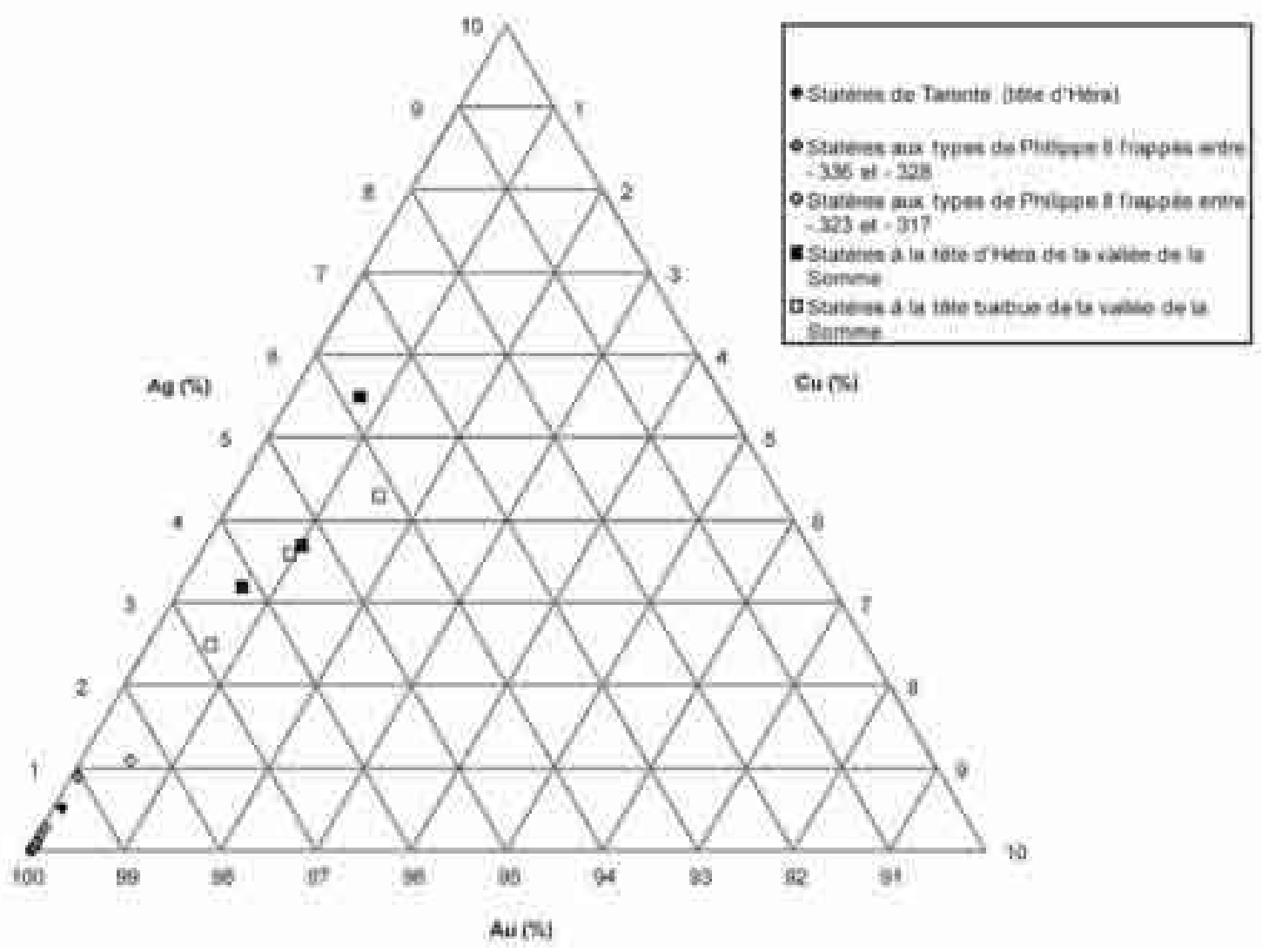

Figure 2 : compositions comparées des prototypes grecs et des imitations de la vallée de la Somme (résultats concernant les statères aux types de Philippe II : Gondonneau, 2001).

Figure 2: compared compositions of Greek prototypes and their Somme valley imitations (results for staters with the types of Philip II: Gondonneau, 2001).

Belgique et dans le Sud de l'Angleterre (Sills, 2003). 14 exemplaires ont été analysés, soit 7 statères et 7 quarts de statère, ce qui n'est certes pas suffisant pour obtenir une vision globale de cette émission importante, mais permet d'ores et déjà de dégager différentes tendances (fig. 3).

La composition des quarts de statère s'inscrit dans la continuité de celle des premiers monnayages de la vallée de la Somme, réunissant toutes les caractéristiques d'un or natif auquel on a ajouté un peu de cuivre : ils sont composés en moyenne de : $82,2 \%$ d'or $( \pm 5,6), 15,3 \%$ d'argent $( \pm 4,6)$, $2,4 \%$ de cuivre $( \pm 1,7)$ et les teneurs en plomb varient entre 17 et $211 \mathrm{ppm}$. On constate néanmoins une évolution : les exemplaires considérés comme étant les plus récents au regard des classements typologiques contiennent davantage d'argent, de cuivre et de plomb. Selon toute vraisemblance, de l'argent a donc été additionné à l'alliage, apportant dans le même temps du plomb, systématiquement associé à l'argent provenant de la galène argentifere. Cette tendance dans la composition de l'alliage monétaire est cohérente avec l'évolution typologique, et accrédite le classement à l'intérieur même d'une série.

La composition des statères au flan large s'inscrit dans une logique différente : ils sont composés en moyenne de : $73,2 \%$ d'or $( \pm 4,4), 22,9 \%$ d'argent $( \pm 2,6), 3,6 \%$ de cuivre $( \pm 2,1)$ et les teneurs en plomb s'échelonnent entre 39 et $543 \mathrm{ppm}$. S'il semble que l'on soit en présence d'un alliage altéré par ajout d'argent et de cuivre, les valeurs de ces additifs demeurent très basses : là encore, l'intérêt d'une altération si modeste ne semble pas évident. Une explication peut être fournie par la comparaison avec la composition des quarts de statère, dans la mesure où celle des plus récents d'entre eux rejoint celle des statères les plus anciens. Il est tentant d'en déduire une chronologie relative entre ces deux dénominations, les quarts de statère apparaissant dès lors comme ayant été frappés à une date antérieure à l'apparition des statères. Cependant, la typologie indique une évolution 


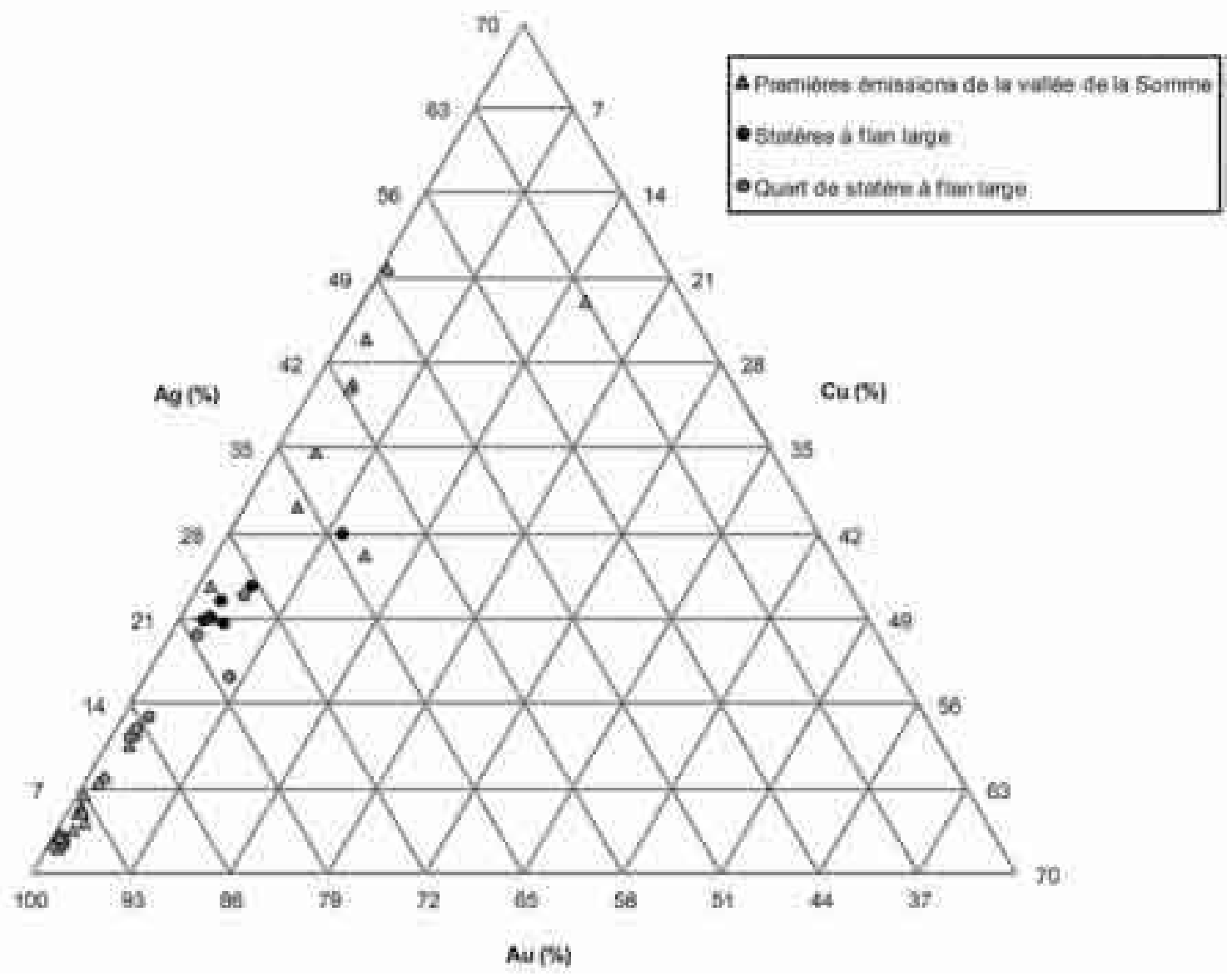

Figure 3 : Composition élémentaire de la série au flan large. Figure 3: Elementary composition of the broad flan series.

similaire et parallèle entre les deux dénominations, ce qui suggère davantage leur contemporanéité. La différence de titre peut aussi s'expliquer par le recours à des sources d'approvisionnement différentes - or natif pour les quarts de statère, refonte d'objets pour les statères - ou encore à deux ateliers distincts pour la frappe de ces deux dénominations.

Les écarts constatés dans le titre des statères et des quarts de statère tendent à confirmer les conclusions déjà formulées pour les premières émissions de la vallée de la Somme quant aux modalités de contrôle du titre ou aux solutions alternatives qui pouvaient lui être substituées (diamètre, poids...).

\section{LES ÉMISSIONS EN OR DU I ${ }^{\mathrm{ER}}$ SIÈCLE AVANT J.-C.}

La dernière phase du monnayage attribué aux Ambiens est représentée par les séries biface et uniface (tableau 1;
11-12), qui se caractérisent par une typologie découlant de la série au flan large mais fondamentalement simplifiée, à tel point que la série uniface ne présente plus aucun type de droit. Ces deux séries sont les plus abondamment frappées. Les cartes de répartition présentent une aire de circulation élargie à toute la Gaule Belgique et le sud de l'Angleterre (Sills, 2003). 10 exemplaires de la série au type biface et 18 statères unifaces ont été analysés (fig. 4).

Ces séries se caractérisent par un titre de plus en plus faible, variant de $81,1 \%$ à $37,7 \%$ d'or. La concentration en cuivre de ces exemplaires, assez importante, implique, comme il a été montré précédemment, un ajout à l'alliage monétaire. Par ailleurs, on constate une forte augmentation des teneurs en plomb, dépassant souvent les 300 ppm (fig. 5), corrélée à l'augmentation parallèle des concentrations en argent, ces deux éléments étant naturellement associés dans la galène argentifère. Ces diverses observations invitent alors à formuler l'hypothèse d'une nette altération 


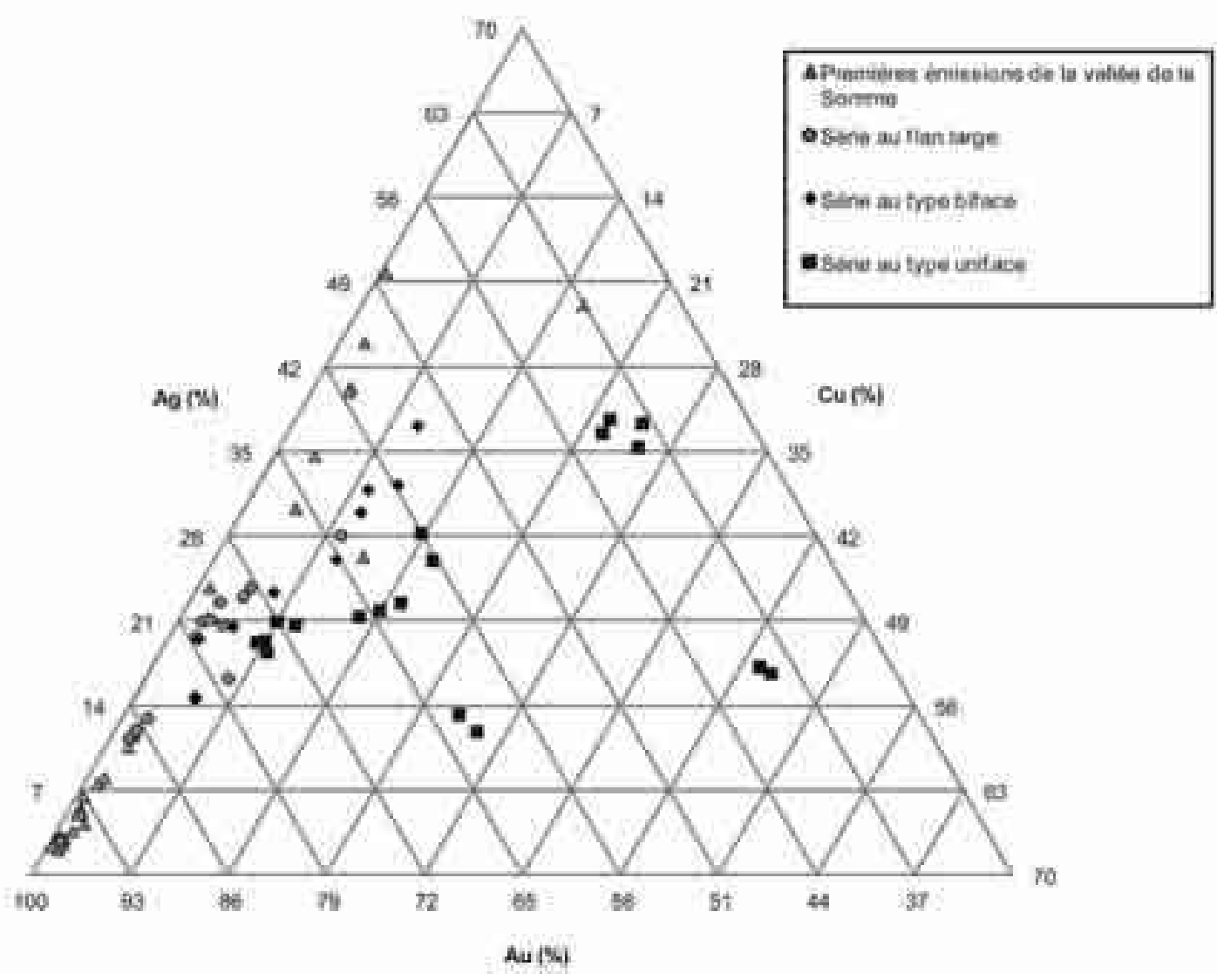

Figure 4 : composition élémentaire des statères aux types biface et uniface.

Figure 4: elementary composition of the biface and uniface series.

de l'alliage monétaire par un ajout intentionnel d'argent et de cuivre.

Ces monnaies sont datées du Irer siècle avant J.-C (Scheers, 1977; Sills, 2003), période marquée par une intensification des échanges économiques et militaires. Ce contexte particulier explique un besoin accru en numéraire et les autorités émettrices ressentent donc la nécessité de produire des émissions abondantes. L'altération de l'alliage monétaire permet alors de multiplier le volume des monnaies frappées sans augmenter la quantité de métal précieux employé.

L'étude de certains éléments traces de la famille des platinoïdes a fourni pour ces séries des résultats intéressants dans la recherche du stock de métal utilisé. On considère généralement que le platine et le palladium, qui sont des éléments "nobles", ne sont pas affectés par les différentes transformations du métal (minéralurgique et métallurgique). Ces teneurs en platinoïdes sont alors utilisées comme indicateur de provenance du métal.

On constate que deux groupes semblent se distinguer au vu des résultats actuels (fig. 6) : le premier (A) rassemble les quarts de statère au flan large et certains statères bifaces et unifaces, signe possible du recours à un même stock d'or, ou d'une refonte des quarts de statère pour permettre la frappe des statères. Le second groupe (B) se caractérise par une augmentation de la concentration en palladium, indépendante des teneurs en platine, qui demeurent assez stables. Cet ensemble B est, dans l'état actuel de la recherche, exclusivement composé de statères du $\mathrm{I}^{\text {er }}$ siècle avant J.-C.

\section{LES MONNAIES UNIFACES EN BRONZE}

On connait par ailleurs des monnaies en bronze présentant les attributs typologiques et le diamètre des statères unifaces (tableau 1; 13). La simplification progressive du type de revers de la série uniface semble trouver son aboutissement avec ces exemplaires en bronze, ce qui conforte la chronologie relative des monnaies unifaces au sein de laquelle ils constituent la dernière classe (Scheers, 1977). Le bronze employé contient en moyenne 11,5\% d'étain. 


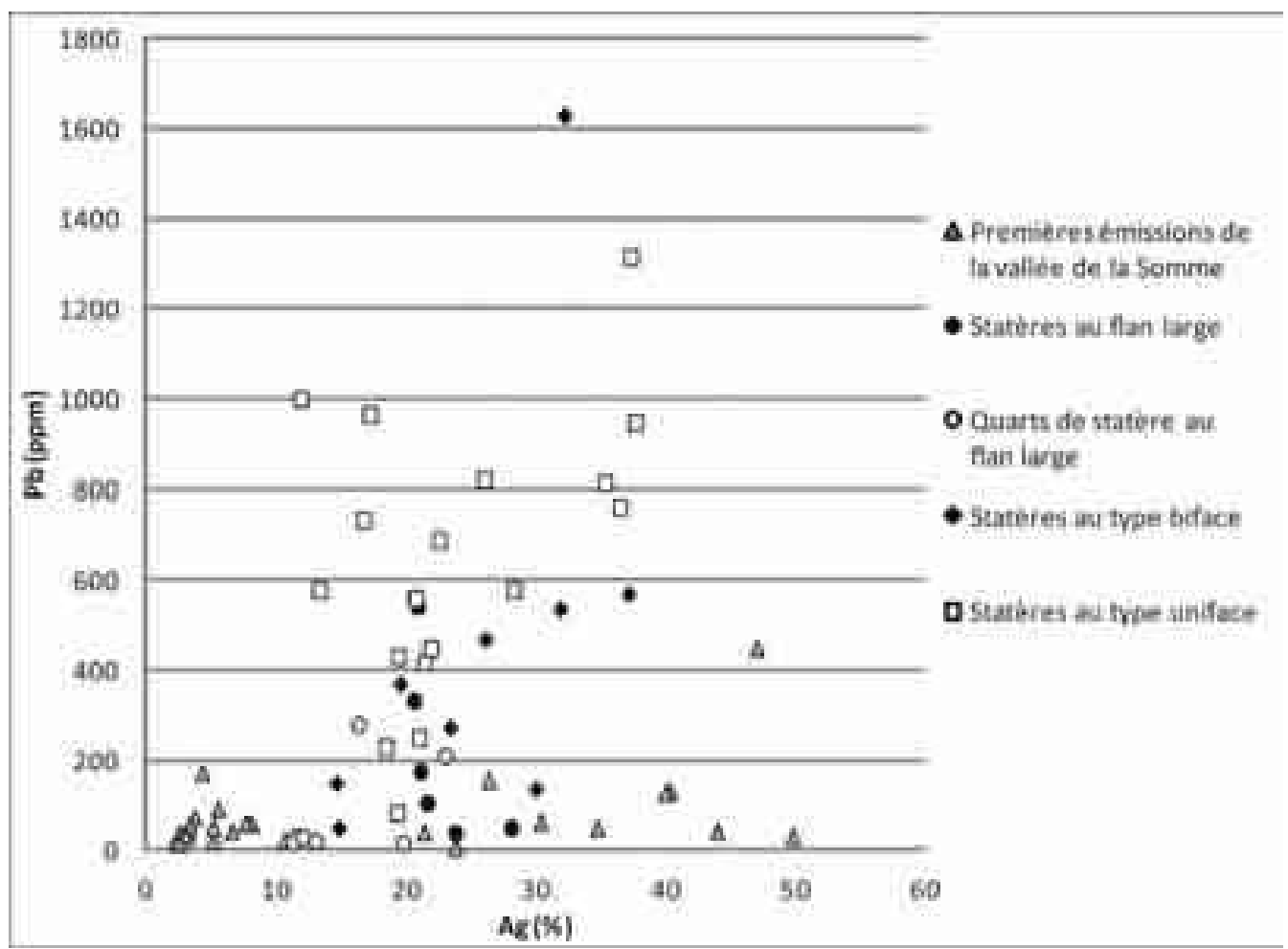

Figure 5 : évolution des teneurs en plomb en fonction de la concentration en argent.

Figure 5 : evolution of lead values according to silver content.

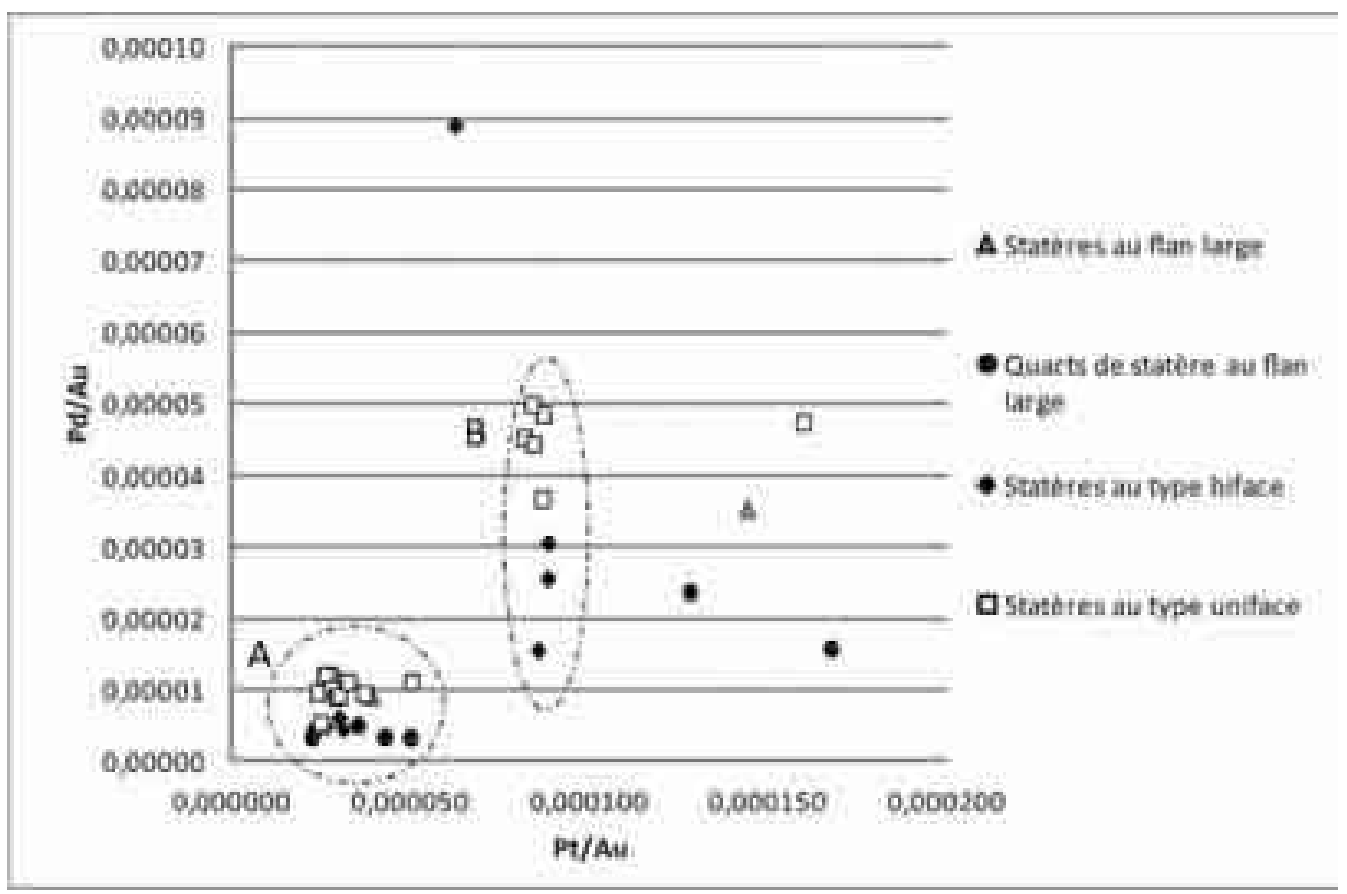

Figure 6 : évolution de la concentration en palladium en fonction des teneurs en platine.

Figure 6: evolution of palladium values according to platinum content. 
Des études effectuées sur cet alliage (Pernot, 1994) semblent suggérer qu'à hauteur de $15 \%$ d'étain, celui-ci s’approche de la couleur de l'or; si la teneur en étain des monnaies unifaces est quelque peu inférieure, la question se pose de savoir si leur couleur approchait celles des monnaies d'or unifaces circulant à la même période. L'altération de l'alliage monétaire influe en effet sur la couleur de l'or, tirant plus ou moins sur le blanc ou le rouge selon les teneurs respectives en argent et en cuivre (fig. 7).

Les statères unifaces les plus récents se composent en moyenne de : $62,6 \%$ d'or $( \pm 0,4), 12,6 \%$ d'argent $( \pm 1,0)$ et $24,7 \%$ de cuivre $( \pm 1,4)$, ce qui leur confère une couleur jaune/jaunâtre. Dans ces conditions, on peut envisager que l'aspect général des exemplaires en bronze devait grandement se rapprocher de celui des derniers statères unifaces frappés. Dans le contexte troublé de la guerre des Gaules, les monnaies unifaces en bronze semblent donc avoir réuni tous les aspects de leurs homologues en or, si ce n'est leur poids, du fait de la moindre densité du bronze. En bref, par-delà l'apparence sans doute proche des exemplaires en or et en bronze, plusieurs questions se posent quant à leur usage : comment ces monnaies de bronze s'inséraient-elles dans le système monétaire en place? Comment étaient-elles perçues par les usagers, qui faisaient nécessairement la différence avec les monnaies d'or du fait de leurs poids respectifs? Ont-elles même été employées en complément du numéraire en or disponible, voire à la place des statères?

\section{ConClusion}

Cette étude a permis de mettre en évidence trois phases consécutives dans la composition des monnaies d'or attribuées aux Ambiens : dans un premier temps, l'alliage monétaire est caractérisé par l'emploi d'un or natif, avec ou sans adjonction de cuivre; l'importante série au flan large se présente ensuite comme une phase de transition durant laquelle est expérimentée l'altération de l'alliage monétaire par l'ajout

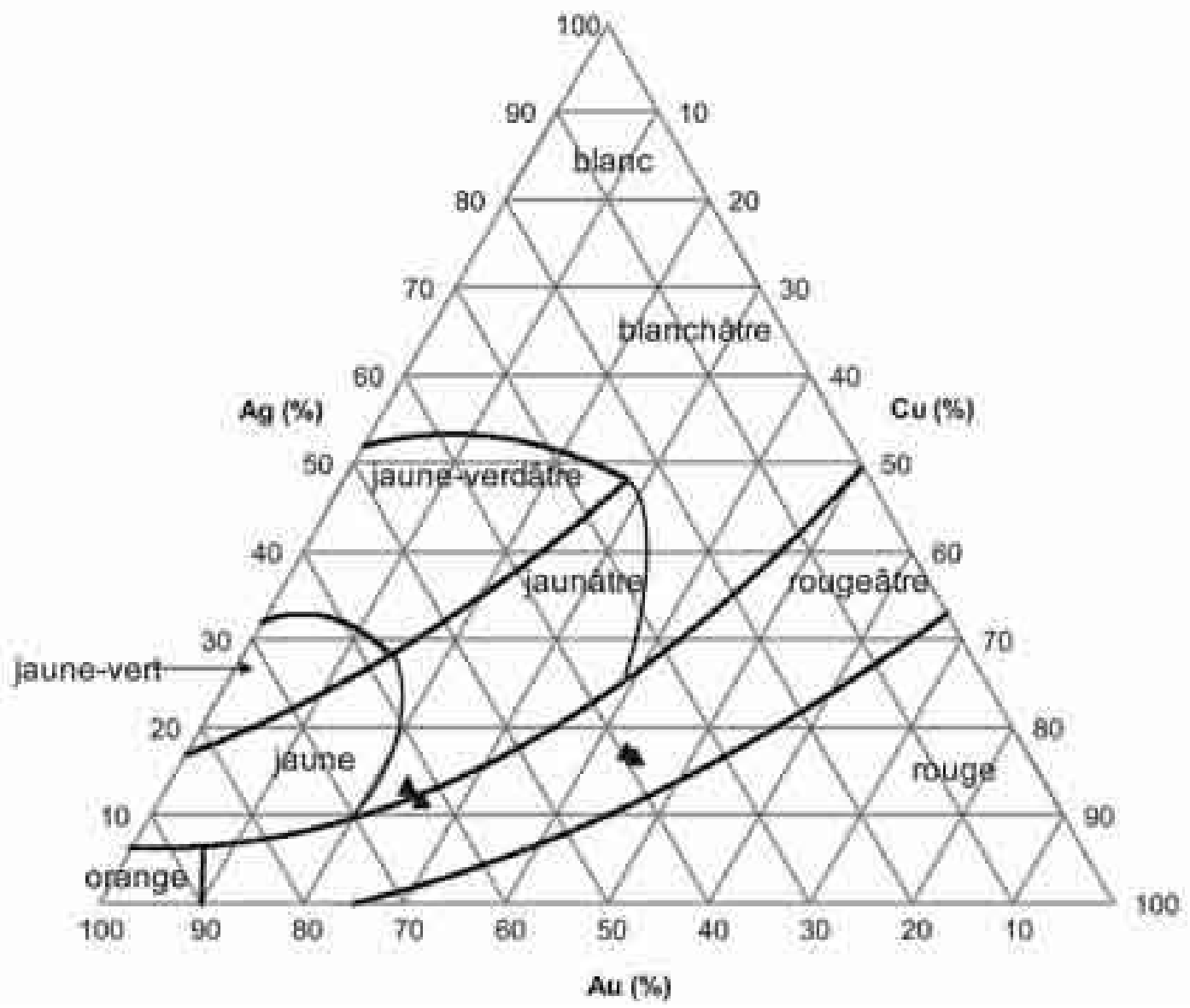

Figure 7 : évolution de la couleur du métal en fonction de la composition de l'alliage.

Figure 7: evolution of metal's colour according to alloy's composition. 
d'argent et de cuivre, dans des proportions encore limitées; enfin, ce procédé est largement étendu, au I ${ }^{\mathrm{er}}$ siècle avant J.-C., pour la frappe des séries biface et uniface, en relation avec une augmentation importante de la production et de la mise en circulation de numéraire.

La succession de ces trois phases apparait cohérente avec les classements établis sur des bases typologiques et métrologiques par Simone Scheers et John Sills, de telle sorte qu'à chacune de ces grandes phases correspondent des caractéristiques typologiques, métrologiques et analytiques propres. Toutefois, au sein de ces trois grandes tendances demeurent des paramètres de variation importants, qui révèlent des situations complexes. Cette étude apporte également la confirmation que les techniques mises en œuvre pour la production monétaire dans cette région ont sensiblement varié selon les périodes et en fonction de circonstances plus difficiles à déterminer : ainsi, les autorités émettrices ont tour à tour eu recours à de l'or natif, ou bien altéré de différentes manières et dans différentes proportions, ajoutant à l'alliage tantôt du cuivre, tantôt de l'argent et du cuivre.

Ces recherches prennent place parmi une série d'études portant sur l'analyse élémentaire des monnayages d'or gaulois, jusqu'alors centrées sur d'autres espaces se rattachant à la Gaule Celtique. À cet effet, elles contribuent à valider, par les enseignements qu'elles fournissent, le principe de l'altération de l'alliage monétaire comme marqueur chronologique des émissions. Toutefois, la spécificité de l'histoire monétaire de la Gaule Belgique se mesure ici à la complexité des résultats obtenus, qui suggère des principes d'organisation économique et des dynamiques spatiales différents de ceux qui prévalent en Gaule Celtique, dans l'état actuel des recherches.

L'importance du monnayage d'or attribué aux Ambiens, ainsi que l'étendue de l'aire au sein de laquelle il a circulé, nous imposent enfin de le considérer au sein de l'espace élargi que constituent la Gaule Belgique et la Bretagne Insulaire. Il s'agit alors de lire sa circulation en parallèle de celle d'autres monnayages d'or de ces régions.

\section{Remerciements}

Les auteurs tiennent à remercier Michel Amandry, Dominique Hollard et Frédérique Duyrat du Cabinet des Médailles de la Bibliothèque nationale de France pour leurs avoir permis d'accéder aux médailliers gaulois et grecs. De plus, nous remercions vivement le laboratoire des Conditions Extrêmes et Matériaux: Haute Température et Irradiation (CEMHTI) auprès duquel ont été réalisées les irradiations des monnaies de bronze.

\section{Bibliographie}

Aubin, G., Barrandon, J.-N., 1994 - Les monnayages armoricains, dans Barrandon, J.-N. (dir.), 1994. L’or gaulois. Le trésor de Chevanceaux et les monnayages de la façade atlantique, Cahiers Ernest-Babelon, 6, p. 141-267.

Barrandon, J.-N. (dir.), 1994 - L’or gaulois. Le trésor de Chevanceaux et les monnayages de la façade atlantique, Cahiers Ernest-Babelon, 6.

Chapman, R. (dir.), 2006 - Microchemical Characterisation of natural gold and artifact gold as a tool for provenancing prehistoric gold artefacts: A case study in Ireland, Applied Geochemistry, 21, p. 904-918.

Gondonneau, A., 2001 - Développement et application des techniques ICP-MS et LA-ICP-MS à la caractérisation de l'or : circulation monétaire en Orient et Occident dans l'Antiquité et au Moyen-âge, Th èse de doctorat, Université Orléans, France.

Gratuze, B., Blet-Lemarquand M. et Barrandon, J.-N., 1994 - Caractérisation des alliages monétaires à base d'or par LA-ICP-MS, Bulletin de la Société Française de Numismatique, p. 163-169.

Kruta, V., 2000 - Les Celtes. Histoire et dictionnaire : des origines à la romanisation et au christianisme, Paris, Robert Laffont ( $1^{\text {re }}$ ed. 1976).

Nieto, S., 2003 - La place du monnayage arverne dans les monnayages gaulois du centre et du sud de la Gaule aux iie et ier siècles avant J.-C., Thèse de doctorat, Université Paris IV-Sorbonne, France.

Pernot,M .,1994- Le bronze, ce n'est pas vert!, dans Actes des IX Journées des Restaurateurs en Archéologie, Paris, CNRS, p. 149161.

Scheers, S., 1977 - Traité de numismatique celtique, la Gaule Belgique, Paris, Les Belles Lettres.

Scheers, S. et Barrandon, J.-N., 1994 - Les imitations du statère de Philippe de Macédoine, dans Barrandon, J.-N. (dir.), 1994. L’or gaulois. Le trésor de Chevanceaux et les monnayages de la façade atlantique, Cahiers Ernest-Babelon, 6, p. 75-139.

SilLs, J., 2003 - Gaulish and early british gold coinage, Londres, Spink. 
Petropoulos, M., Pontrandolfo, A. and Rizakis, A.D., 2006 - Quinta campagna di ricognizioni archeologiche in Egialea (settembre-ottobre 2006). Annuario della Scuola Archeologica Italiana di Atene, 84: 941-964.

Price, S. and Nixon, L., 2005 - Ancient Greek agricultural terraces: evidence from texts and archaeological survey. American Journal of Archaeology, 109: 665-694.

Rachкам, O., 1972 - Appendix III. Charcoal and plaster impression. In P.M. Warren (dir.). Myrtos: an Early Bronze Age settlement in Crete. British School of Athen, suppl. 7, London, 283-298.

Renfrew, A.C., 1972 - The emergence of civilization: the Cyclades and the Aegean in the third millennium BC. Methuen and Co., London.

Renfrew, J.M. 1973 - Paleoethnobotany. The prehistoric food plants of the Near East and Europe. Methuen and Co., New York.

Riba, M., 1997 - Effects of cutting and rainfall on pattern on resprouting vigour of Erica arborea L. Journal of Vegetation Science, 8, (3): 401-404.

Runnels, N.C. and Hansen, J., 1996 - The olive in the prehistoric Aegean: the evidence for domestication in the Early Bronze Age. Oxford Journal of Archaeology, 15, (1): 299-308.

Santoriello, A., Scelza, F., and Bove, R. - Egialea Survey Project: Method and Strategies, in Beyond the Artefact. Procedings of the 32th Computer Applications and Quantitative Methods in Archaeology conference in Prato, Italy, April 2004), British Archaeological Reports, in press.

SchweIngruber, F.H., 1990 - Microscopic wood anatomy. Swiss Federal Institute for Forest, Birmensdorf.

Sfikas, G., 1983 - Arbres et arbustes sauvages de la Grèce. Efstathiadis Group, Athens.

Simpson, R.H., 2007 - Interdisciplinary survey in Messenia, Southwest Peloponnese, Greece. Geoarchaeology: a international journal, 22, (1): 111-120.

Sutton, S.B., 2000 - Contingent countryside: settlement, economy and land use in southern Argolid since 1700. Stanford University Press, Standford.

Stummer, A. 1911 - Zur Urgeschichte der Rebe und des Weinbaues. In Mitteilungen Der Anthropologischen Gesellschaft in Wein XLI: 283-296.
Tan, K., Iatrou, G. And Johsen, B., 2001 - Endemic plants of Greece: the Peloponnese, Vol. 1. Gads Forlag, Copenhagen.

Terral, J.F. and Arnould-Simard, G., 1996 - Beginnings of olive cultivation in relation to Holocene bioclimatic changes. Quaternary Research, 46, (2): 176-185.

Terral, J.F., 2000 - Exploitation and management of the olive tree during prehistoric times in Mediterranean France and Spain. Journal of Archaeological Science, 27, (2): 127-133.

Terral J.F., Alonso N., Buxò i Capdevila, R., Chatti, N., Fabre, L., Fiorentino, G., Marinval, P., Pèrez Jorda, G., Pradat, B., Rovira, N. and Alibert, P., 2004 - Historical biogeography of olive domestication (Olea europaea L.) as revealed by geometrical morphometry applied to biological and archaeological material. Journal of Biogeography, 31, (1), 63-77.

Tomaselli, R., 1977 - The degradation of the Mediterranean maquis. Ambio 6, (6): 356-362.

Trabaud, L., 1991 - Fire regimes and phytomass growth dynamics in a Quercus coccifera garrigue. Journal of Vegetation Science 2, (3): 307-314.

Tsiouvaras, C.N., 1987 - Ecology and management of kermes oak (Quercus coccifera L.). Shrublands in Greece: A Review. Journal of Range Management, 40, (6): 542-546.

Verroios, G. and Georgiadis, T., 2011 - Aleppo pine forests of northern and western Peloponnisos (southern Greece): Plant communities and diversity. Plant Biosystems, 145, (3): 608619.

Wells, B. and Runnels, C.N., 1996 - The Berbati-Limnes archaeological survey, 1988-1990. Svenska Institutet i Athen, Stockholm.

Zohary, D. and Hopf, M., 2000 - Domestication of plants in the Old World. The origin and spread of cultivated plants in West Asia, Europe, and the Nile Valley. Oxford University Press, Oxford. 